\title{
The HiSCORE concept for gamma-ray and cosmic-ray astrophysics beyond $10 \mathrm{TeV}$
}

\author{
Martin Tluczykont ${ }^{\mathrm{a}, *}$, Daniel Hampf ${ }^{\mathrm{a}}$, Dieter Horns ${ }^{\mathrm{a}}$, Dominik Spitschan ${ }^{\mathrm{a}}$, Leonid Kuzmichev ${ }^{\mathrm{b}}$, \\ Vasily Prosin $^{\mathrm{b}}$, Christian Spiering ${ }^{\mathrm{c}}$, Ralf Wischnewski ${ }^{\mathrm{c}}$ \\ a Institute for Experimental Physics, University of Hamburg, Luruper Chaussee 149, 22761 Hamburg, Germany

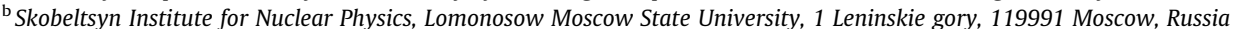 \\ ${ }^{\mathrm{c}}$ DESY, Platanenallee 6, 15378 Zeuthen, Germany
}

\section{A R T I C L E I N F O}

\section{Article history:}

Received 19 July 2013

Received in revised form 20 February 2014

Accepted 10 March 2014

Available online 19 March 2014

\section{Keywords:}

Cosmic rays

Gamma-rays

Instrumentation

Air Cherenkov astronomy

Pevatrons

\begin{abstract}
A B S T R A C T
Air-shower measurements in the primary energy range beyond $10 \mathrm{TeV}$ can be used to address important questions of astroparticle and particle physics. The most prominent among these questions are the search for the origin of charged Galactic cosmic rays and the so-far little understood transition from Galactic to extra-galactic cosmic rays. A very promising avenue towards answering these fundamental questions is the construction of an air-shower detector with sufficient sensitivity for gamma-rays to identify the accelerators and large exposure to achieve accurate spectroscopy of local cosmic rays. With the new ground-based large-area (up to $100 \mathrm{~km}^{2}$ ) wide-angle $(\Omega \sim 0.6-0.85 \mathrm{sr}$ ) air-shower detector concept HiSCORE (Hundred*i Square-km Cosmic ORigin Explorer), we aim at exploring the cosmic ray and gamma-ray sky (accelerator-sky) in the energy range from few $10 \mathrm{~s}$ of $\mathrm{TeV}$ to $1 \mathrm{EeV}$ using the non-imaging air-Cherenkov detection technique. The full detector simulation is presented here. The resulting sensitivity of a HiSCORE-type detector to gamma-rays will extend the energy range so far accessed by other experiments beyond energies of $50-100 \mathrm{TeV}$, thereby opening up the ultra high energy gamma-ray (UHE gamma-rays, $E>10 \mathrm{TeV}$ ) observation window.
\end{abstract}

(c) 2014 Elsevier B.V. All rights reserved.

\section{Introduction}

The current knowledge about the origin of cosmic rays has been accumulated following two different approaches: (i) by measuring in detail the energy spectrum and mass composition of the local cosmic-ray population and (ii) by gamma-ray $(E>100 \mathrm{MeV})$ observations of both individual astrophysical objects as well as the diffuse emission from the interstellar medium. Both approaches provide complementary information/constraints on the most relevant quantities: e.g. the measurement of spallation products and cosmo-genic nuclei provides information on the energy dependence of cosmic-ray transport and the escape time of cosmic rays out of the Galaxy. Gamma-ray observations constrain the spatial distribution and properties of the cosmic ray accelerators and the density of cosmic rays in the interstellar medium.

Cosmic-ray measurements through air-shower techniques are the only means to collect sufficient event statistics to measure cosmic rays at energies close to the knee $\left(\approx 3 \times 10^{15} \mathrm{eV}\right)$ in the

\footnotetext{
* Corresponding author. Tel.: +49 408998 2993; fax: +49 4089982170.

E-mail address: martin.tluczykont@physik.uni-hamburg.de (M. Tluczykont).
}

all-particle energy spectrum. The traditional air-shower detectors sample the lateral density function (LDF) of secondary particles or photons on the ground. Given the large intrinsic fluctuations in the shower development and that only a small fraction of the particles are sampled $\left(\approx 10^{-4}\right)$, the energy resolution and sensitivity to different primary particles is rather limited. Combining detection of different components of the air shower as e.g. realized in the KASCADE air shower field [1], improves the situation considerably but suffers from limited collection area. Established techniques to follow the longitudinal air shower development include muon tracking, air Cherenkov, and air fluorescence observations.

The latter technique has been realized quite early and remains one of the most sensitive techniques at ultra-high energies [Linsley, Fly's eye, HiRes, Pierre-Auger Observatory, TelescopeArray, see [2], and references therein]. The non-imaging air Cherenkov technique measures the arrival time and the LDF of the Cherenkov photons in the air shower front. This technique is sensitive to the longitudinal air shower development (mainly position of the shower maximum) as demonstrated with e.g. Themistocle [3], AIROBICC [4], Blanca [5], Tunka [6], Jakutsk [7]. The longitudinal air shower development is sensitive to the initial 
particle species. Both techniques allow a comparably good energy resolution which suffers less from the fluctuations and the limited sampling. A number of new approaches for air shower detection have been proposed and partially tested including long-wavelength $(\mathrm{MHz})$ radio measurements ${ }^{1}$ see e.g. [8], and molecular Bremsstrahlung emission at $\mathrm{GHz}$ frequencies $[9,10]$. In the sense of shower-front sampling, the long-wavelength radio observations are comparable to the air Cherenkov technique while the molecular Bremsstrahlung has analogies to the air fluorescence (mostly isotropic emission) and would allow for imaging of the air shower development.

For approach (i) - spectroscopy and measurement of chemical composition of cosmic rays in the energy range from below the knee to the ankle $\left(10^{18} \mathrm{eV}\right)$ - the air Cherenkov approach appears to be among the best choices, considering its good energy resolution of the order of $10 \%$, and a typical resolution of the shower maximum of the order of $30 \mathrm{~g} / \mathrm{cm}^{2}$ [6]. See also Section 3.3. For approach (ii) - gamma-ray observations - the currently most successful technique is the imaging air Cherenkov technique with multiple telescopes (imaging air Cherenkov telescopes: IACTs). A large array of IACTs is currently under design to achieve a ten-fold improvement in flux sensitivity as compared to current generation instruments: the Cherenkov Telescope Array CTA, see [11]. Nevertheless CTA is designed to achieve optimum sensitivity at $\mathrm{TeV}$ energies and will suffer from its limited collection area at energies beyond $100 \mathrm{TeV}$. The non-imaging air Cherenkov technique allows to extend the collection area to several square kilometers with a moderate number of read-out channels. ${ }^{2}$ In combination with the demonstrated good angular resolution in non-imaging Cherenkov air shower arrays, a multi-km² array with good sensitivity above $10 \mathrm{TeV}$ appears feasible and is explored here.

With the Hundred $* \mathrm{i}$ Square-km Cosmic ORigin Explorer HiSCORE, we want to cover both approaches (i) and (ii) described above. A central question will be the search for the elusive pevatrons [13], the accelerators of cosmic rays up to the PeV energy regime. For more details on physics topics for HiSCORE, see [14] and references therein.

\section{HiSCORE detector design}

\subsection{Detector array layout}

HiSCORE will consist of an array of wide-angle light-sensitive detector stations, distributed over an area of the order of $100 \mathrm{~km}^{2}$. As compared to previous experiments, important aspects of HiSCORE are different (see Table 1): an instrumented area larger by more than an order of magnitude, up to a factor 16 larger lightcollecting area per station, and the usage of fast $\mathrm{GHz}$ waveform sampling electronics.

Since we aim at a very large instrumented area, a low array density with large inter-station spacings is favoured. Fig. 1 shows the lateral photon density function (LDF) of Cherenkov light on the ground. Within a radius of $120 \mathrm{~m}$ around the shower core position, the LDF is roughly constant. Beyond $120 \mathrm{~m}$, the photon density decreases following a power law. With a station spacing of $100 \mathrm{~m}$ or more (depending on the array layout and partly variable), HiSCORE will primarily measure the outer part of the LDF, i.e. most stations will sample the LDF beyond $120 \mathrm{~m}$ distance from the shower core. Still, a few stations will lie within the central $120 \mathrm{~m}$ of the lightpool. Due to the low Cherenkov photon density far away from the shower core, a large light collection area $a$ of the individual

\footnotetext{
${ }^{1}$ The dominant emission processes are geo-synchrotron and charge separation.

2 There are complementary approaches using large field of view cameras which would allow to increase the spacing of individual telescopes [12].
}

Table 1

Basic design characteristics of the HiSCORE (highlighted in bold face) detector in comparison with other experiments. The total instrumented area $A$, the light collection area $a$ of an individual station, the field of view FoV, the inter-station distance $d$ and the number of detector stations $N$ are listed.

\begin{tabular}{lllllr}
\hline Parameter: & $A$ & $a$ & FoV & $d$ & $N$ \\
Unit & {$\left[\mathrm{km}^{2}\right]$} & {$\left[\mathrm{m}^{2}\right]$} & {$[\mathrm{sr}]$} & {$[\mathrm{m}]$} & \\
HiSCORE & $\mathbf{1 0 0}$ & $\mathbf{0 . 5}$ & $\mathbf{0 . 6 0 - 0 . 8 5}$ & $\mathbf{1 5 0}^{\mathrm{a}}$ & $\mathbf{4 4 8 9}$ \\
Tunka-133 & $1^{\mathrm{b}}$ & 0.031 & 1.8 & 85 & 133 \\
Blanca & 0.2 & 0.1 & 0.12 & 35 & 144 \\
AIROBICC & 0.04 & 0.13 & 1 & $15-30$ & 49 \\
Themistocle & 0.08 & 0.5 & 0.008 & $50-100$ & 18 \\
\hline
\end{tabular}

a Inter-station spacing used for the simulation results presented in the present paper are not optimized yet.

b In 2011, the effective area for high energy events was increased to $3 \mathrm{~km}^{2}$ by extending the array with additional 42 optical detectors, placed at a distance of $1 \mathrm{~km}$ from the array center [6].

detector stations is needed. With the standard array configuration and a chosen area $a=0.5 \mathrm{~m}^{2}$, on average 3 stations within $120 \mathrm{~m}$ of the shower core are found to be above threshold for showers at $50 \mathrm{TeV}$ primary energy. The energy threshold for gamma-rays at reconstruction level therefore is $50 \mathrm{TeV}$. When using alternative layouts with partly higher station densities or larger PMTs (see Section 4), the energy threshold at reconstruction level can be reduced towards our aim of $10 \mathrm{TeV}$. At $100 \mathrm{TeV}$, the LDF can be sampled in the power law part up to core distances of $450 \mathrm{~m}$. In Fig. 1, the basic difference in scale becomes apparent: The inter-station spacing of the HiSCORE array is of the same order of magnitude as the total side-length of the AIROBICC detector.

\subsection{Detector station}

A HiSCORE detector station consists of four photomultiplier tubes (PMTs), each equipped with a light-collecting Winston cone of $\approx 30^{\circ}$ half-opening angle pointing to the zenith. The advantages of using four PMT channels per station are on the one hand the suppression of random triggers from nightsky background (NSB) light through a local coincidence trigger condition, and on the other hand an increase of the light collecting station area $(a)$. When using four $8^{\prime \prime}$ PMTs and a height of $0.5 \mathrm{~m}$ of the Winston cones $a=0.5 \mathrm{~m}^{2}$ is achieved. A schematical view of the station concept is given in Fig. 2.

The PMTs have to fulfill two basic requirements: the operational gain must be such that the anode current stays within manufacturer limits under the expected NSB conditions. With a nominal gain of $10^{4}$, the 6-stage PMT KB9352 from Electron Tubes fulfills this requirement. A modified R 5912 Hamamatsu PMT with six dynodes is an interesting alternative. The dynamic range has to be as high as $10^{5}$, since we aim at measurements between $10 \mathrm{TeV}$ and $1 \mathrm{EeV}$. This could be achieved by reading out one or two dynodes in addition to the anode signal. Assuming a voltage range of the readout of $14 \mathrm{mV}$ to $1 \mathrm{~V}$ (e.g. DRS4 evaluation board, [15]), the anode (high gain, first readout stage) channel provides a dynamic range of 70 . At the upper end of this voltage range, the actual anode signal is $100 \mathrm{mV}$ (when using a preamplification of a factor 10), i.e. well within the linear anode voltage range which extends up to roughly $2 \mathrm{~V}$. With a dynode (low gain, second readout stage) channel at a factor 50 lower amplification, sufficient overlap between both channels is given. The total dynamic range then is 3500 . An additional, second dynode readout would provide a further extension of the dynamic range up to $10^{5}$. Alternatively, without a third readout stage, events at the highest energies could also be reconstructed using stations far away from the shower core, and applying appropriate low weights for the inner (saturated) stations. While such 


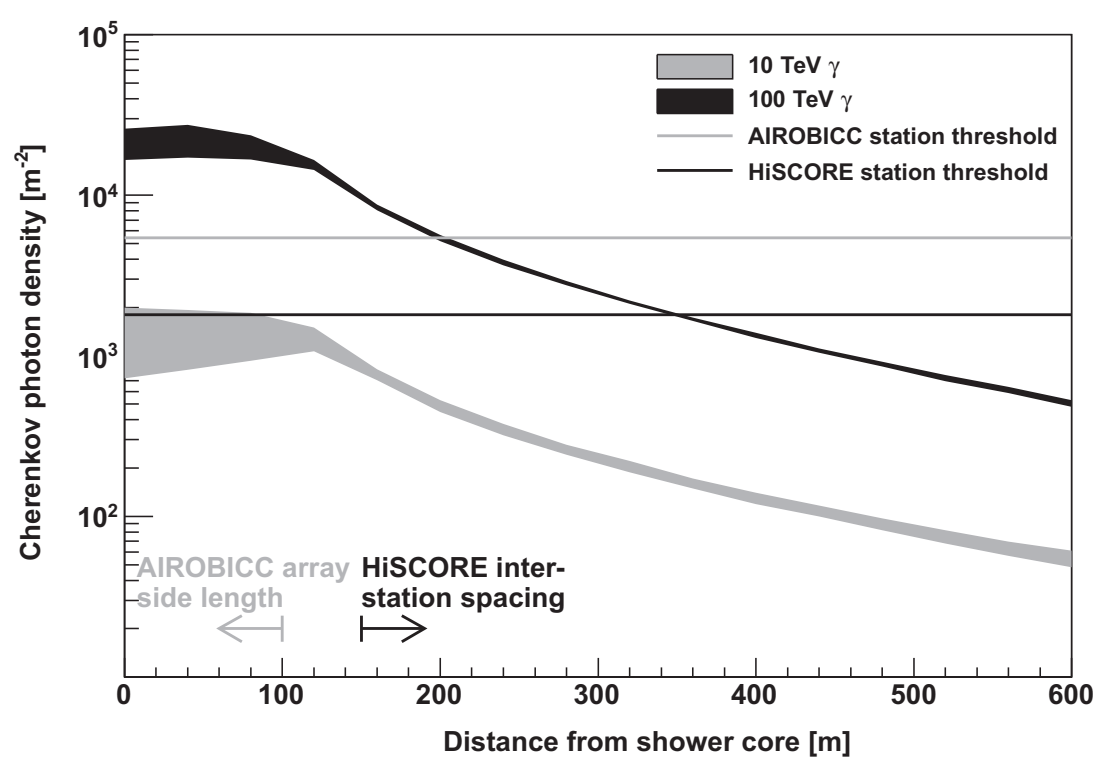

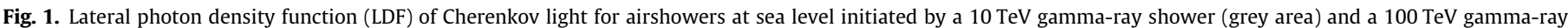

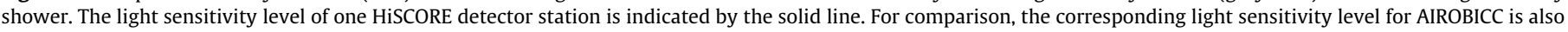
shown (grey line). Note, the observation level of AIROBICC was $2200 \mathrm{~m}$ with a correspondingly higher photon density.

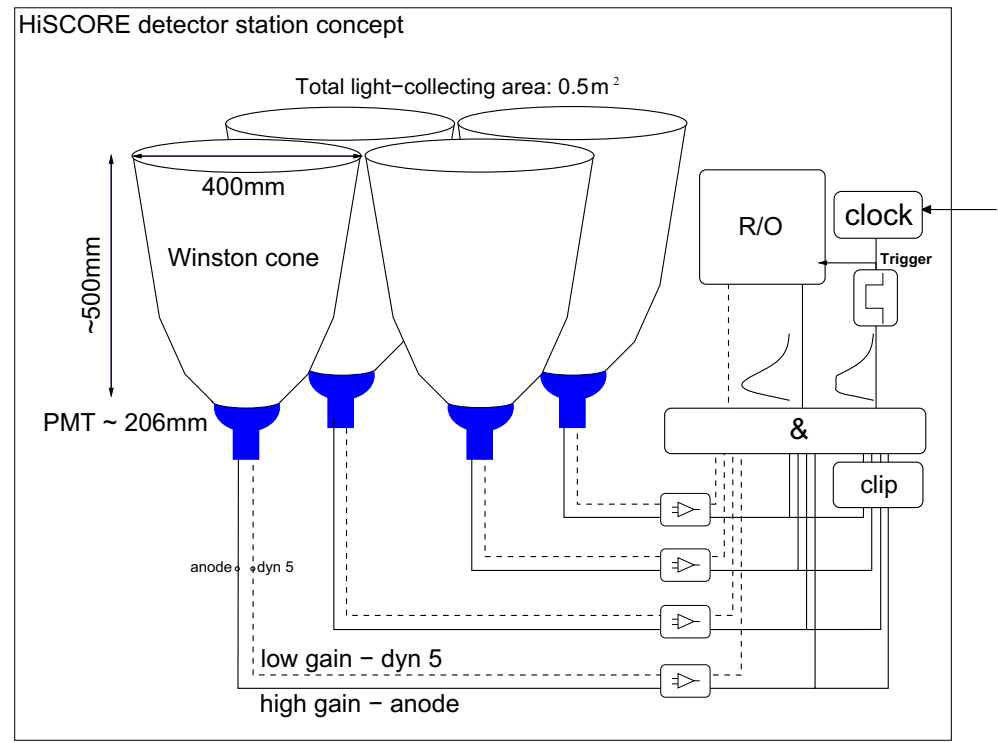

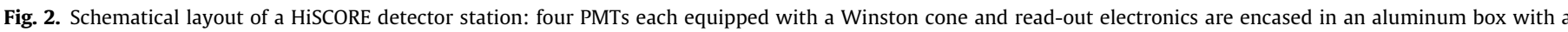
sliding lid (not shown). To increase the dynamic range, the PMT anode and the next to last dynode are read out.

methods of increasing the dynamic range are feasible, they will require careful calibration and will ultimately be a source of systematic errors towards the high energy end of the sensitive detector range.

The Winston cones can be conveniently built from segments of reflective aluminum layer on a synthetic, flexible carrier material (Alanod 4300UP). Ten segments are cut out from this material and assembled similarly to a barrel. The Winston cone shape is well reproduced along the optical axis.

The inside of the station box is equipped with slow-control electronics, a lid motor, high-voltage (HV) systems, a local station trigger, and a read-out system (pre-amplifiers, analog signal sampling board). A fast signal read-out and digitization in the $\mathrm{GHz}$ regime is provided by a read-out board with up to 8 channels based on the DRS 4 chip [15], with a depth of 1024 cells at a resolution of up to $0.2 \mathrm{~ns}$ per cell $(5 \mathrm{GHz})$. In order to retain a sufficiently wide read-out window, a sampling frequency of $1 \mathrm{GHz}$ is used corresponding to a read-out window of $1 \mu \mathrm{s}$.

For a large array such as HiSCORE, with large inter-station spacings, accurate relative timing is crucial. Simulations show that a relative timing accuracy between stations of the order of $1 \mathrm{~ns}$ is required to optimize the angular resolution as well as the reconstruction accuracy of the position of shower maximum. Systems for relative synchronization at the (sub)-ns accuracy level are currently under study. Possible approaches are WhiteRabbit (PTP over synchronous ethernet [16]), with sub-ns resolution reached in the HiSCORE prototype [17-19], or a system based on the ethernet carrier frequency such as used by the Tunka-133 array [20], or radio signal phase synchronization [21]. 


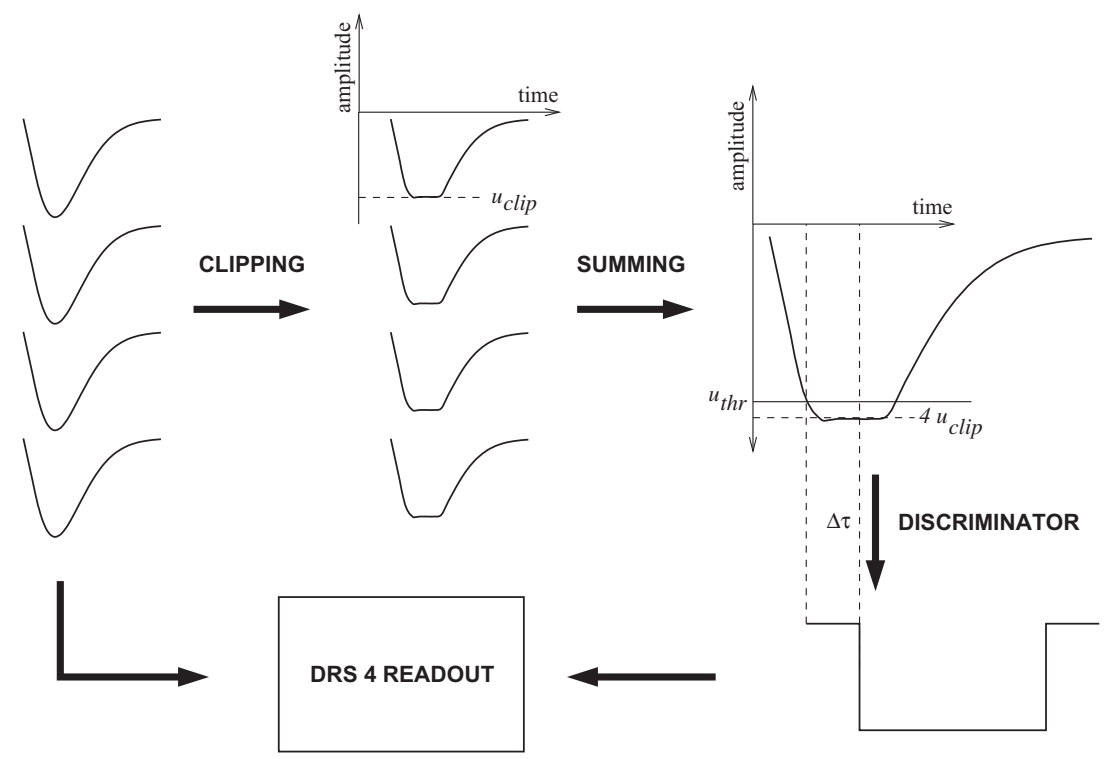

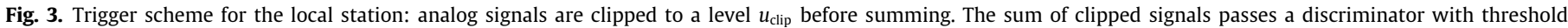
$0<u_{\text {thr }} / u_{\text {clip }}<4$, which finally triggers the DRS 4 read-out.

\subsection{Trigger and read-out}

The station trigger is illustrated in Fig. 3 (also see Fig. 2). The analog PMT signals are clipped to a pre-defined amplitude level $u_{\text {clip }}$ and then summed. The clipped sum then passes a discriminator set to a threshold level $u_{\text {thr }}$, with $0<\left|u_{\text {thr }}\right| /\left|u_{\text {clip }}\right|<4$. The exact value of the ratio $u_{\text {thr }} / u_{\text {clip }}$ is a free parameter. In the simulation presented here, this ratio was set to 3.8. A local station trigger is issued when the time-over-threshold of the clipped sum is larger than $\Delta \tau$. The clipped sum trigger prevents false triggers from large signal fluctuations in individual PMTs, therewith suppressing afterpulses, NSB photons, and triggers from uncorrelated cosmicray muons (see also Section 3.2), allowing a lower station trigger threshold. The value of $\Delta \tau$ depends upon the choice of the discriminator. Here, $\Delta \tau=7 \mathrm{~ns}$ was used. At each local station trigger, the data are read out and sent to a central PC farm. Additionally, all neighboring stations which have not issued a trigger are read out as well. In the simulated array setup (150 m grid constant), a typical gamma-ray event with $200 \mathrm{TeV}$ primary energy triggers of the order of 10 stations. A distribution of photon counts in the simulated array is shown in Fig. 4 for a simulated gamma-ray event at $187 \mathrm{TeV}$. Stations that issue a local trigger in the simulation are additionally marked with a grey circle.

\section{HiSCORE simulation results}

\subsection{Simulation of air showers and detector response}

\subsubsection{Simulation software}

Air showers were simulated with CORSIKAv675 [22] using the hadronic interaction models QGSJET01c.f [23], and GHEISHA [24], and the electromagnetic interaction model EGS4. The systematic error on the background estimation is due to the limited accuracy of hadronic interaction models, and to the lack of precise knowledge of the chemical composition of hadrons. While the former will be improved with models modified on the basis of LHC data [25], the latter is one result to be deduced from HiSCORE data in the future. Gamma rays, protons, helium- nitrogen- and iron-nuclei were simulated in the energy range from $10 \mathrm{TeV}$ to $5 \mathrm{PeV}$

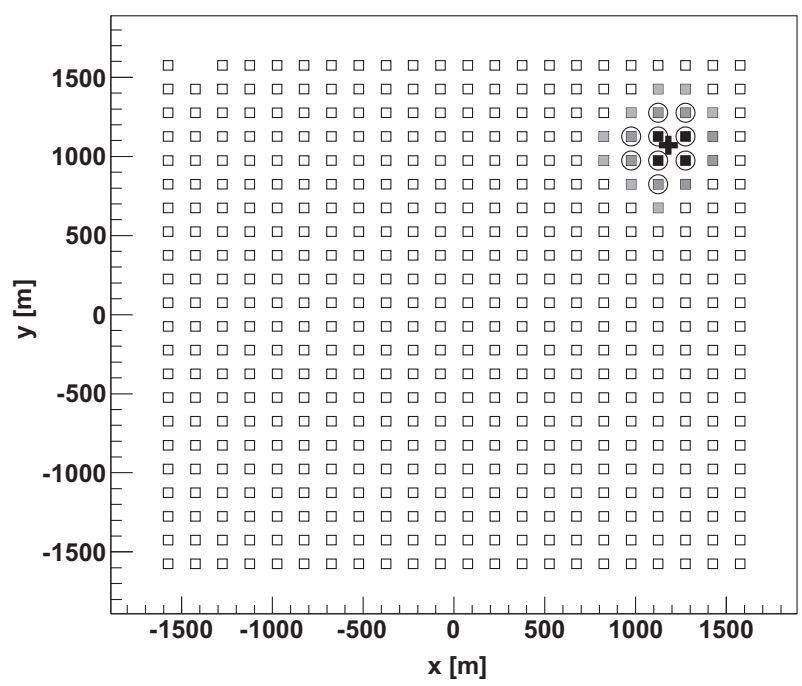

Fig. 4. HiSCORE event display of a $187 \mathrm{TeV}$ gamma-ray event recorded by the standard geometry array configuration. Recorded photons in the detector stations are binned in high (black), medium (dark grey) and low (light grey) densities. Triggered stations are marked by an additional circle. The cross indicates the simulated position of the shower core.

following a power law distribution $d N / d E \propto E^{-1}$ resulting in equal numbers of events per decade. Additionally, protons from $5 \mathrm{TeV}$ to $10 \mathrm{TeV}$ were simulated for the estimation of the detector trigger rate (see Section 3.2). Due to our focus to high energies, we require a large Cherenkov light pool. Thus, the detector array was simulated at an altitude of $0 \mathrm{~m}$ above sea-level. Simulations at higher altitudes show that a benefit at low energies is only achieved when using a smaller inter-station spacing, and thus a smaller overall instrumented area [26]. Within CORSIKA, the IACT option [27] was used, storing Cherenkov photons in spheres of $1 \mathrm{~m}$ radius at sea-level, each sphere representing one detector station. Different geometrical array layouts were simulated. The standard array layout consists of 484 stations distributed over a regular square grid as shown in Fig. 4. The standard array covers a total instrumented area of roughly $10 \mathrm{~km}^{2}$ (3.15 km side length). Each station in the standard configuration was simulated based on $8^{\prime \prime}$ PMTs as 
described above. Air showers were simulated with uniformly distributed impact position in a square with side length $3.8 \mathrm{~km}$, corresponding to a simulated area of $14.44 \mathrm{~km}^{2}$. The directions were simulated randomly with a uniform density in solid angle up to a zenith angle of $30^{\circ}$.

A full detector simulation (sim_score) was implemented on the basis of the IACT package [27]. At the position of each CORSIKA sphere, a detector station with 4 PMT-channels is simulated in sim_score. In detail, sim_score comprises the following elements:

- Winston cone acceptance tables based on ray-tracing simulations [28].

- Atmospheric scattering of the Cherenkov photons is calculated using MODTRAN [29].

- PMT quantum efficiency (wave-length dependent) as in ElectronTubes data sheet for KB9352 (29\% at maximum).

- Overall PMT photoelectron collection efficiency of $90 \%$.

- PMT signal pulse shape (see inlay of Fig. 5) using a parametrization by [30, see Appendix A].

- Station trigger: the clipped sum station trigger as in Fig. 3.

- The NSB photon baseline is simulated separately and added to the signals at read-out level (pulse shaping and afterpulses are included in this simulation).

An interval of $2 \mathrm{~s}$ of NSB noise is simulated as an array of photons uniformly distributed over time, and corresponding to a constant flux of $3 \times 10^{12}$ photons $/\left(\mathrm{m}^{2} \mathrm{sr} \mathrm{s}\right)$. An average quantum efficiency for NSB photons of 0.1 (calculated using the wavelength dependent quantum efficiency of the PMTs and a spectrum for NSB photons), and a constant photo electron conversion efficiency of 0.9 were used. Each photo electron is stored in a read-out array (each array element corresponding to a 1 ns DRS 4 cell), according to the shape and amplitude response described above. A $1 \mu$ s interval of simulated NSB noise after subtraction of the average baseline is shown in Fig. 5, along with two signals corresponding to 200 and 500 photoelectrons (p.e). The results presented in this paper were obtained using a clipped sum threshold corresponding to 180 p.e $\left(u_{t h r}\right.$, see Fig. 3).

\subsection{Trigger rates}

The relevant contributions to the trigger rate are night-sky background (NSB) photons, and cosmic rays. In the following paragraphs, we present estimations of these rates, and show that the flux of uncorrelated atmospheric muons does not contribute.

\subsubsection{Hadron trigger rates}

The differential trigger rate $R(E)$ of an array can be calculated in the following way:

$R(E)=\sum_{\mathrm{Z}=1}^{92} \Phi_{Z}(E) \cdot \int d \Omega A_{\mathrm{eff}}(i \in\{\mathrm{p}, \mathrm{L}, \mathrm{M}, \mathrm{H}\}, E, \vartheta, \varphi)$,

where the sum runs over the individual cosmic-ray fluxes $\Phi_{i}(E)$ of the elements as provided by [31] and the effective areas for cosmic ray nuclei have been derived for representative elements: Helium for the light ( $L: Z=2-5$ ), Nitrogen for the medium ( $M: Z=6-24$ ), and iron for the heavy $(\mathrm{H}: \mathrm{Z}>24)$ groups. The effective area is given as the ratio of triggered to simulated events multiplied by the simulated area. The effective area $A_{\text {eff }}$ depends in principle on the zenith angle $\vartheta$ as well as on the azimuth angle $\varphi$. The simulations show however, that up to $\vartheta \approx 25^{\circ}$, the effective area does not vary strongly which justifies a simplified treatment: the effective area is assumed to be constant over $\vartheta$ and the effective solid angle within the constant region $\left(\vartheta<25^{\circ}\right)$ is $\Delta \Omega=0.6 \mathrm{sr}$. In Fig. $6, A_{\text {eff }}(i, E)$ is given for gamma-rays and the 4 hadronic particle types $(\mathrm{H}, \mathrm{He}, \mathrm{N}$, $\mathrm{Fe}$ - corresponding to protons, light, medium and heavy groups), requiring only one station to trigger at a single station threshold of 180 p.e. and using the trigger scheme described in Fig. 3.

A parametrization of the cosmic ray spectrum [31] is weighted with the corresponding effective areas to calculate the expected trigger rates for hadronic cosmic ray events. The resulting trigger rate as a function of energy for an individual HiSCORE station is shown in Fig. 7. The integral single-station local trigger rate is found to be $12.8 \mathrm{~Hz}$. The hadron trigger rate of the full $10 \mathrm{~km}^{2}$-array of 484 stations is $1.77 \mathrm{kHz}$ (p: $875 \mathrm{~Hz}, \mathrm{~L}: 505 \mathrm{~Hz}, \mathrm{M}: 290 \mathrm{~Hz}, \mathrm{H}$ : $100 \mathrm{~Hz})$.

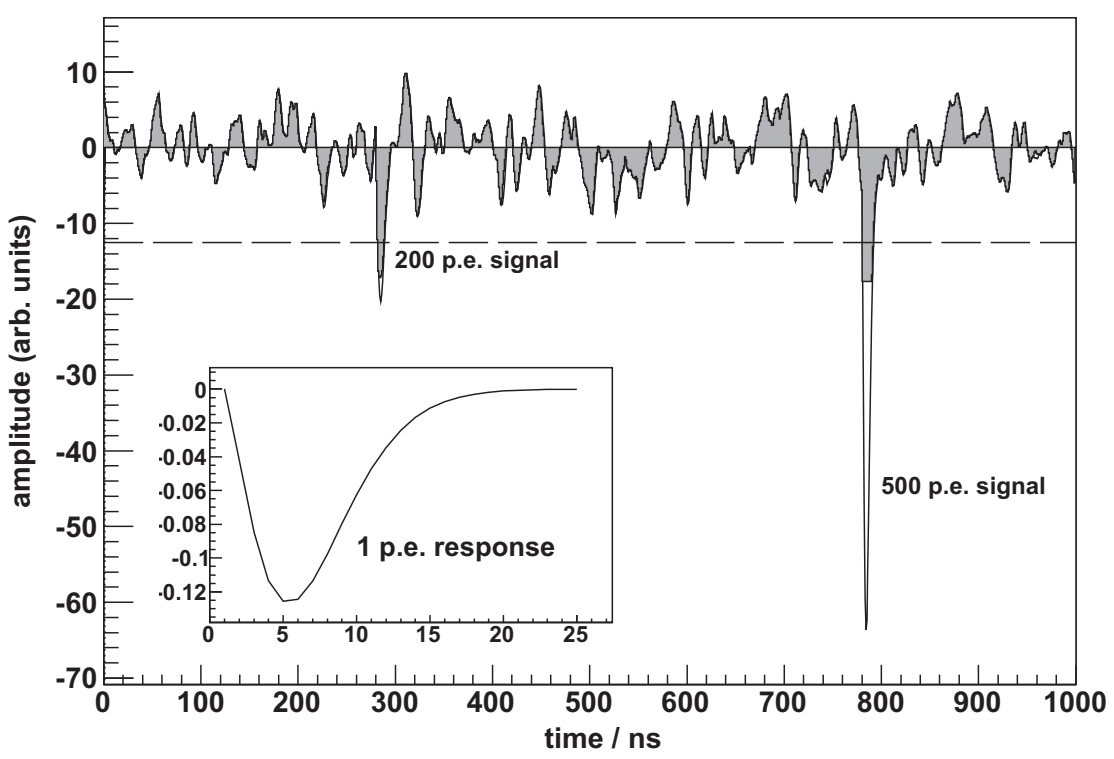

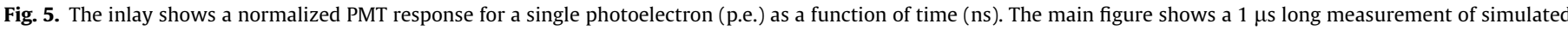

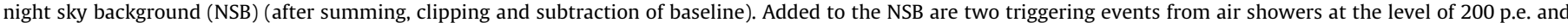

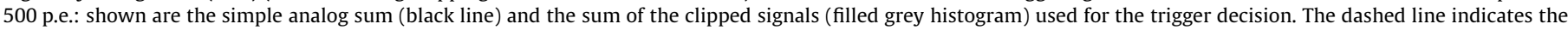
trigger threshold $u_{t h r}$ used here. 


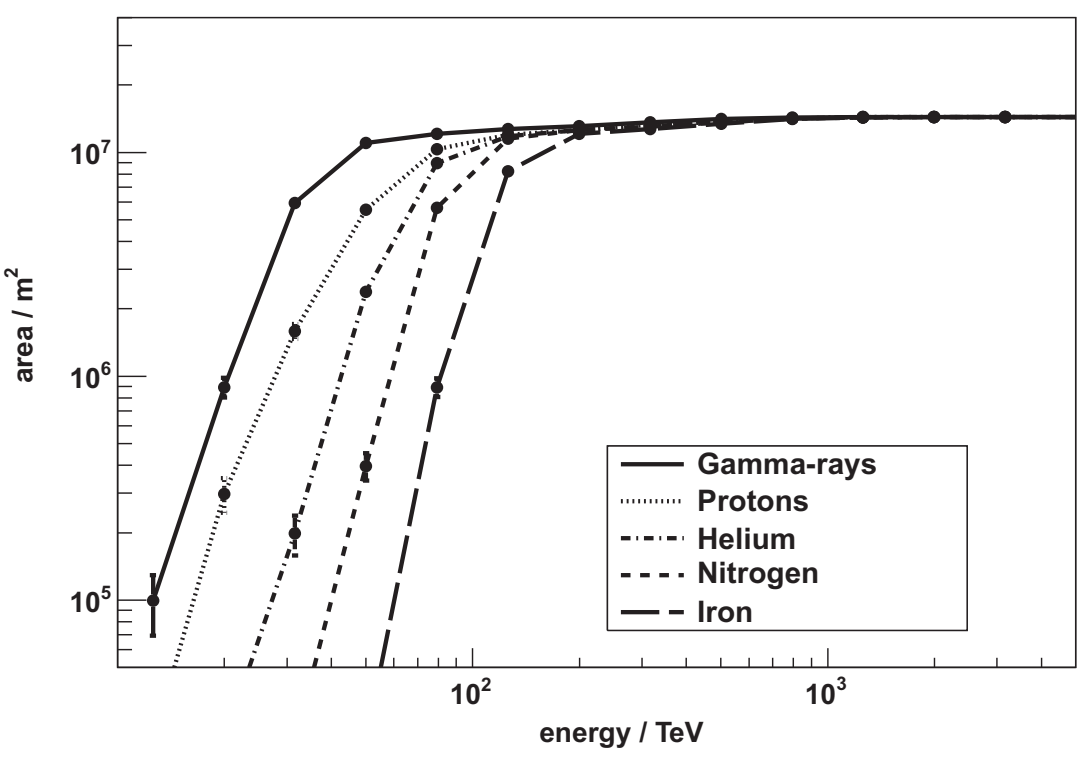

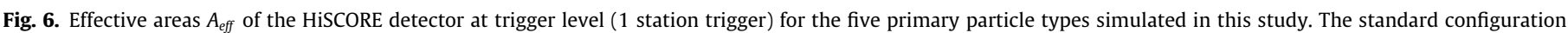
presented here results in an average number of 3 triggered stations for a $50 \mathrm{TeV}$ gamma-ray.

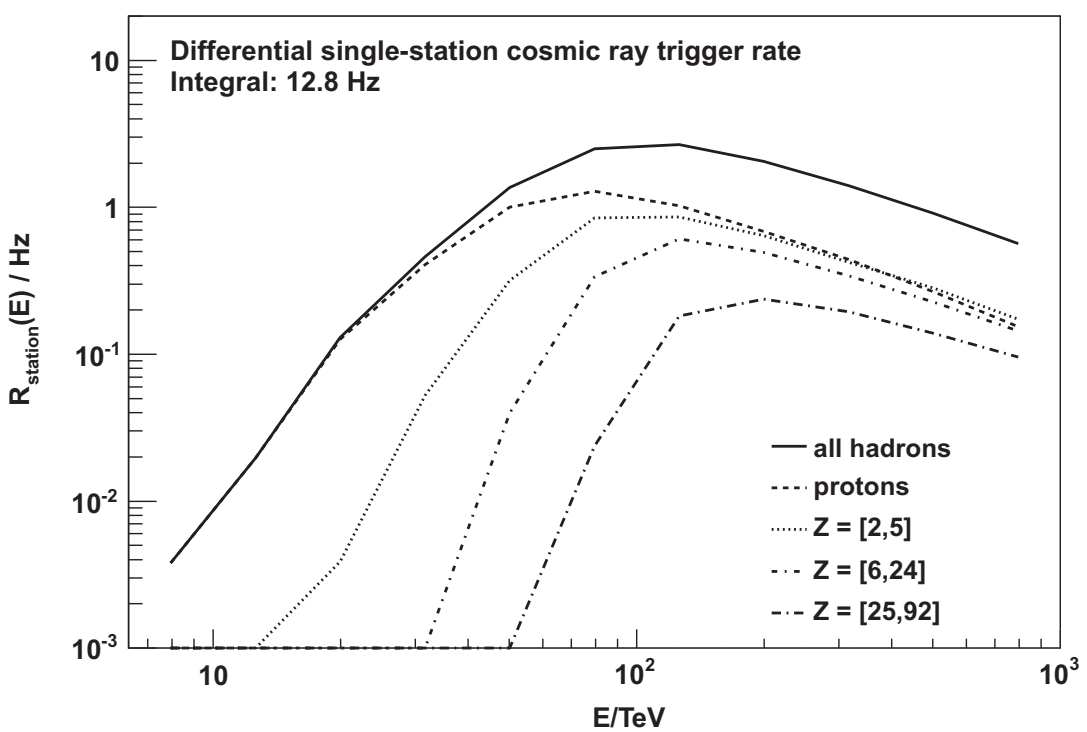

Fig. 7. Differential all-hadron trigger rate for one single HiSCORE station. A local station trigger threshold of 180 p.e. was used.

\subsubsection{Night-sky background trigger rates}

The night-sky background (NSB) is induced by light sources outside of the atmosphere including direct and scattered starlight, scattered moon light, zodiacal light and light produced within the atmosphere including air glow and human-made light sources. The expected trigger rate induced by NSB photons was simulated using a dedicated simulation of a full 4-channel station including the pulse shaping and trigger scheme. Measurements of the NSB level at Fowlers gap in Australia [32] were used as input for these simulations. An NSB-induced single-station local trigger rate of $100 \mathrm{~Hz}$ was found at a clipped sum threshold of 180 p.e., demonstrating that the single-station trigger rate is clearly dominated by NSB photons (see Fig. 8). A reduction of the data flow could be achieved when also using a second trigger level, requiring coincident ( $\mu$ s-window) triggers of 2 neighboring stations. This could be realized on software level and is not necessary on hardware level as long as the total data flow from single-station triggers can be handled (e.g. using a central PC farm). Alternatively, slightly increasing the station discriminator threshold to 190 p.e. results in an NSB trigger rate of $50 \mathrm{~Hz}$ without significantly affecting the sensitivity, and only raising the energy threshold (linearly) by $5 \%$.

\subsubsection{Uncorrelated cosmic ray muon trigger rate}

The overall flux of vertical incidence atmospheric muons measured at sea level is 160 particles $\mathrm{m}^{-2} \mathrm{~s}^{-1}$ [33]. Taking into account the detector properties, the total average signal from one muon in one station is of the order of 100 p.e. The trigger rate for uncorrelated muons as shown in Fig. 8 was calculated with the full simulation chain (CORSIKA \& sim_score), using different clipped sum thresholds. As can be seen from Fig. 8, the muon trigger rate is found to be negligible in comparison with the rate of NSB photon induced triggers. The rate was calculated for a trigger setup with a clipped sum threshold of 180 p.e. and $\Delta \tau=7 \mathrm{~ns}$. 


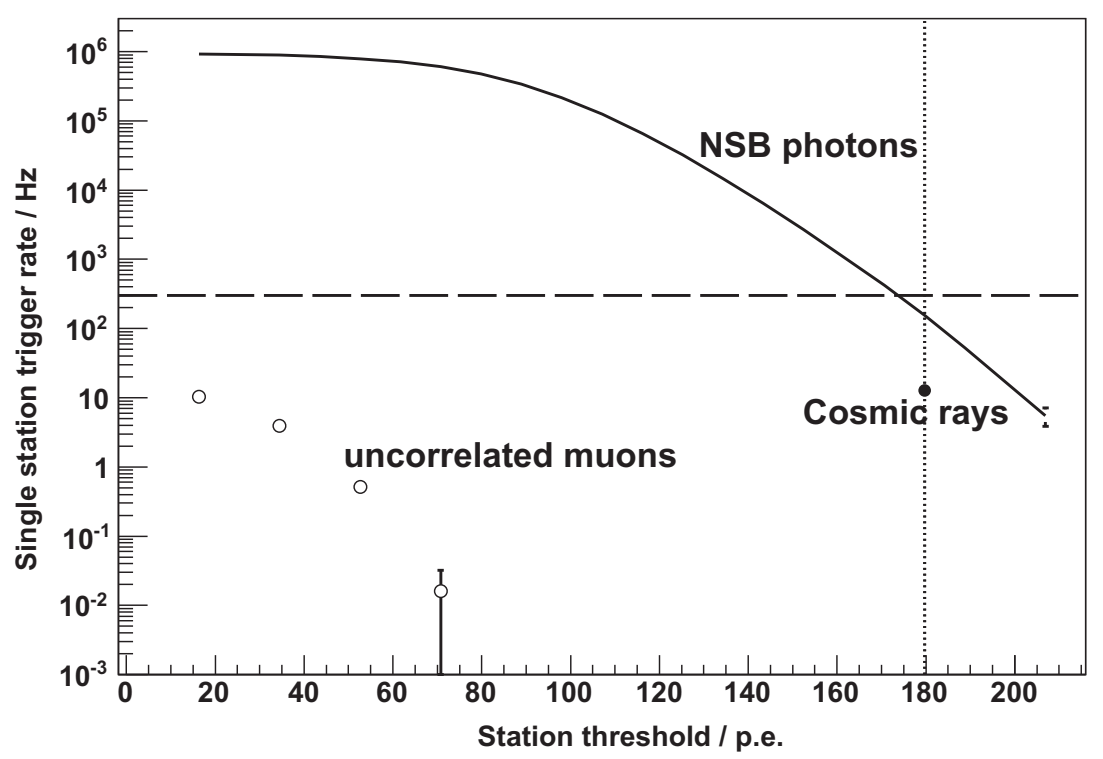

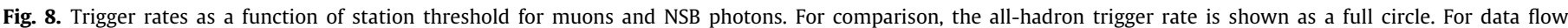
minimization, we aim at a maximum trigger rate of $300 \mathrm{~Hz}$, as indicated by the dashed line.

\subsection{Air shower reconstruction}

Air shower reconstruction algorithms for HiSCORE were introduced in [34,28]. The arrival direction is reconstructed using an analytical model for the arrival time of Cherenkov photons at the detector stations. The primary particle energy is reconstructed from the light density value at a fixed distance from the shower core, as reconstructed from the lateral density function of a shower event. As shown in [34], the standard array configuration achieves an angular resolution of $0.25^{\circ}$ at $100 \mathrm{TeV}$ and $0.1^{\circ}$ at $1 \mathrm{PeV}$. The achieved energy resolution is $20 \%$ at $100 \mathrm{TeV}$, improving to better than $10 \%$ at $1 \mathrm{PeV}$. The resolution of the height of the shower maximum is $70 \mathrm{~g} / \mathrm{cm}^{2}$ at $50 \mathrm{TeV}$ and reaches $40 \mathrm{~g} / \mathrm{cm}^{2}$ at $1 \mathrm{PeV}$. These numbers, even though obtained with very basic reconstruction algorithms, are comparable to the energy and shower maximum resolutions of the Tunka-133 array (15\% energy-, and $25 \mathrm{~g} / \mathrm{cm}^{2}$ shower maximum resolution at higher energies) [6]. Primary particle identification is done on the basis of the reconstructed energy and shower height. At the energy threshold and up to $100 \mathrm{TeV}$, the gamma-hadron separation is inefficient (quality factor of 1.0). Beyond $100 \mathrm{TeV}$, the quality factor gradually improves to 2.0 at $1 \mathrm{PeV}$. A comprehensive discussion can be found in [28]. The quality of spectroscopic measurements of the all-particle cosmic ray

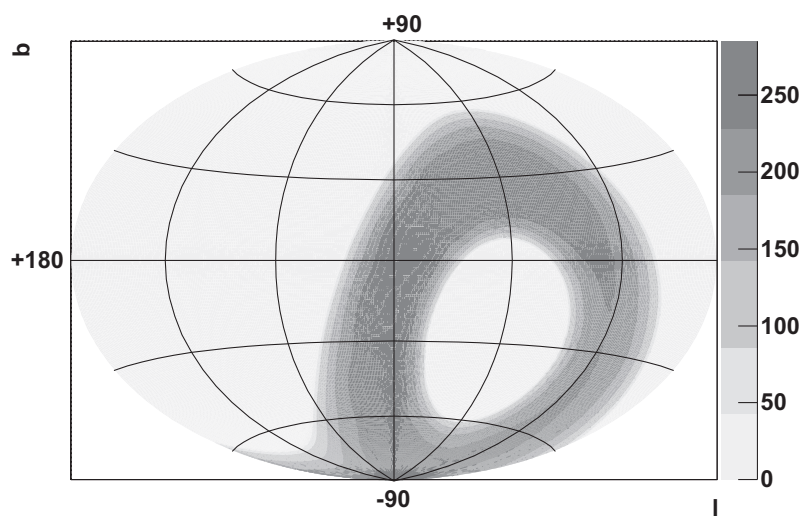

Fig. 9. Exposure in hours for one year of data taking at a southern site $\left(31^{\circ}\right.$ southern geographical latitude), using only events within $25^{\circ}$ half opening angle. An area of one $\pi \mathrm{sr}$ is covered for more than $200 \mathrm{~h}$ within one observation year. spectrum will depend on the energy resolution and the total effective area of the instrument. At 10\% energy resolution and an instrumented area of $100 \mathrm{~km}^{2}$, HiSCORE will allow a high accuracy for spectroscopic reconstruction of cosmic rays around the knee region and beyond.

\subsection{Sensitivity to gamma-rays}

The point-source survey sensitivity (for a definition see below) to gamma-rays was calculated on the basis of the simulation and reconstruction described above.

HiSCORE always operates in survey mode, i.e. all objects which are visible during darkness time within the visible cone of the instrument are observed. In the standard observation mode, the optical axis of the detector stations points to the zenith. The observation time for any given position in the sky was calculated for an observation site at $31^{\circ}$ southern geographical latitude. The accumulated exposure time for a celestial position is derived using an acceptance cut taking into account only events within $25^{\circ}$ half opening angle [26]. While the region of the sky covered is restricted by the detector site, the large effective field of view of $0.6 \mathrm{sr}$ leads to a total sky coverage of $\pi \mathrm{sr}$ in one year at an

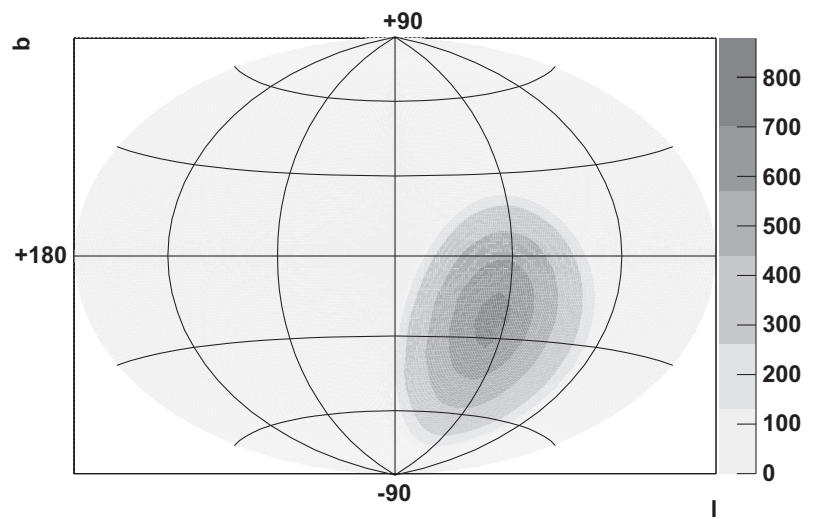

Fig. 10. Exposure in hours for one year of data taking in a $30^{\circ}$ tilted south mode, at a southern site ( $31^{\circ}$ southern geographical latitude), using only events within $25^{\circ}$ half opening angle. 


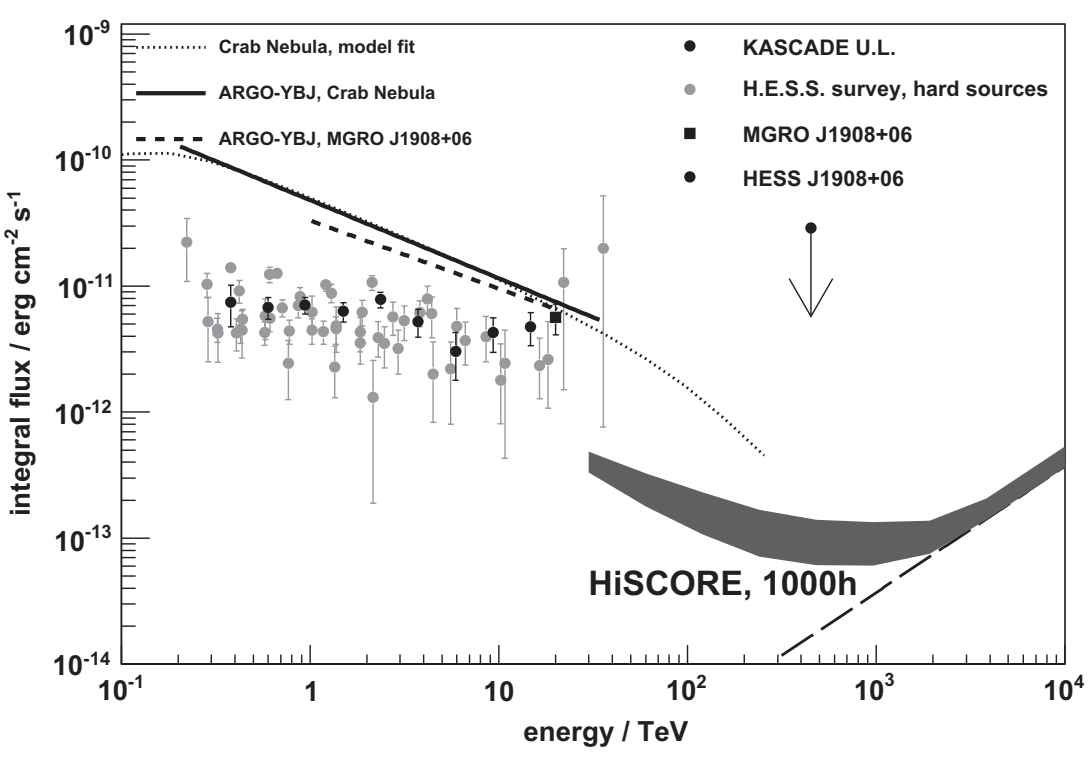

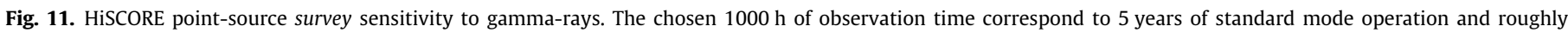

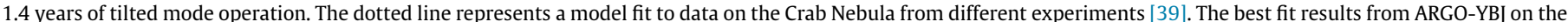

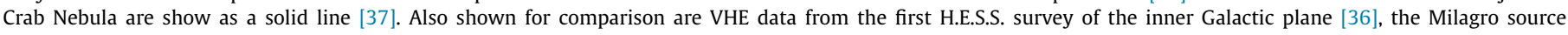
MGRO J1908+06 (Milagro data [40], H.E.S.S. data [41], ARGO-YBJ data [42]), and an upper limit from KASCADE [1].

exposure depth ranging from $200 \mathrm{~h}$ to $283 \mathrm{~h}$, as illustrated in Fig. 9. For comparison, the first survey of the inner Galaxy with H.E.S.S. covered a total solid angle of the order of $0.1 \mathrm{sr}$ at an exposure level of the order of $10 \mathrm{~h}$. The amount of survey time that can be allocated with a pointing instrument (IACT) depends on the time dedicated for deep exposure of individual objects, which might have precedence over a survey. The advantage of IACTs is their flexibility when determining the exact region to survey. The situation is different for HiSCORE. The region that is surveyed is defined by the region covered by the (fixed) instrument within a year. We plan to improve this situation using a reorientation of the detectors along their north-south axis (tilting), allowing to access different regions of the sky. This way, after a given operation time in standard observation mode (e.g. few years without tilting), the total sky coverage can be increased, by consecutively tilting the detector stations to the south (tilted south observation mode) and to the north for additional operation periods of a few years. For illustration, using a tilting angle of $30^{\circ}$ towards the south (for a southern observation site), the sky covered is extended to the region shown in Fig. 10, with a significantly deeper exposure per source within this region. We used a total observation time of $1000 \mathrm{~h}$, corresponding to 5 years of standard mode operation and roughly 1.4 years of tilted mode operation.

A minimum of $5 \sigma$ detection significance ${ }^{3}$ and 50 gamma-rays were required to define the flux sensitivity. The background numbers were calculated in an analogous way as described in Section 3.2 using however effective areas after quality cuts [34]. The resulting point-source sensitivity for $100 \mathrm{~km}^{2}$ instrumented area is shown in Fig. 11. The dark shaded area shows the range of sensitivities achieved when using pessimistic (upper bound) and optimistic (lower bound) assumptions [34, also see]. The pessimistic bound was obtained by using a conservative alpha factor (ratio of solid angles of source to background measurements in Eq. 9 of Li and Ma [35]) of $\alpha=1$ and an angular gamma-ray point source cut (the size of the source region around a test position) efficiency of 0.68 . In the optimistic scenario, $\alpha \ll 1$. Additionally, the gamma-ray efficiency of the point source cut was set to 1 in the background free regime, i.e. above 2.1 PeV. Also shown in Fig. 11 are representative flux mea-

\footnotetext{
${ }^{3}$ The significance is calculated using Eq. 9 of [35].
}

surements from H.E.S.S. [36], ARGO-YBJ [37], Milagro [38], and an upper limit from KASCADE [1].

At the energy threshold, the sensitivity of HiSCORE is mainly limited by the angular resolution and secondarily by the gammahadron separation (See Section 3.3). At the upper energy end, the sensitivity is limited by count statistics and depends linearly on the inverse of the product of total detector area and the exposure time, forming the background free regime (straight black dashed line in Fig. 11). In the central energy regime, the sensitivity is limited by the gamma-hadron separation. Here, we only show the sensitivity for the standard layout. Improvements of the angular resolution or to the gamma-hadron separation (large PMTs, graded array, muon detectors, see Section 4) are not included. Such detector enhancements will cause the background free regime to extend to lower energies, effectively pushing down the sensitivity curve toward the straight black dashed line. With HiSCORE, it will be possible to study the continuation of the spectra of known Galactic sources up to several $100 \mathrm{TeV}$. In this context, it will be important to investigate whether some of these sources could be cosmic ray pevatrons [13], [14,43, also see].

A comparison of the sensitivity of the HiSCORE standard configuration to point-source sensitivities of other gamma-ray experiments (CTA [11,44], HAWC [45], and LHAASO [46,47], the latter being valid for an exposure of one calendar year, and adapted to a minimum statistics requirement of 50 events) is shown in Fig. 12. The sensitivity of IceCube for detecting a $5 \sigma$ excess of neutrinos after 4.5 years of observation from stacked gammaray sources as explained in Gonzalez-Garcia et al. [48] is also included. Here, the underlying assumption is that the entire gamma-ray emission is of hadronic origin. For comparison of sensitivities, it is important to note that the sensitivity shown for HiSCORE is the point-source survey sensitivity for $\pi$ sr of the sky. While the shown sensitivities for HAWC, LHAASO, and IceCube are also valid for surveys, the CTA sensitivity is given for pointed observations of $50 \mathrm{~h}$ in a small field of view of the order of $\pi / 100$ sr (dashed line), for a survey of $\pi$ sr (upper bound of grey area) and a survey of the Galactic plane (lower bound of grey area). Furthermore, it has to be noted that the total observation time available per year to HiSCORE depends on the observation mode (see above). 


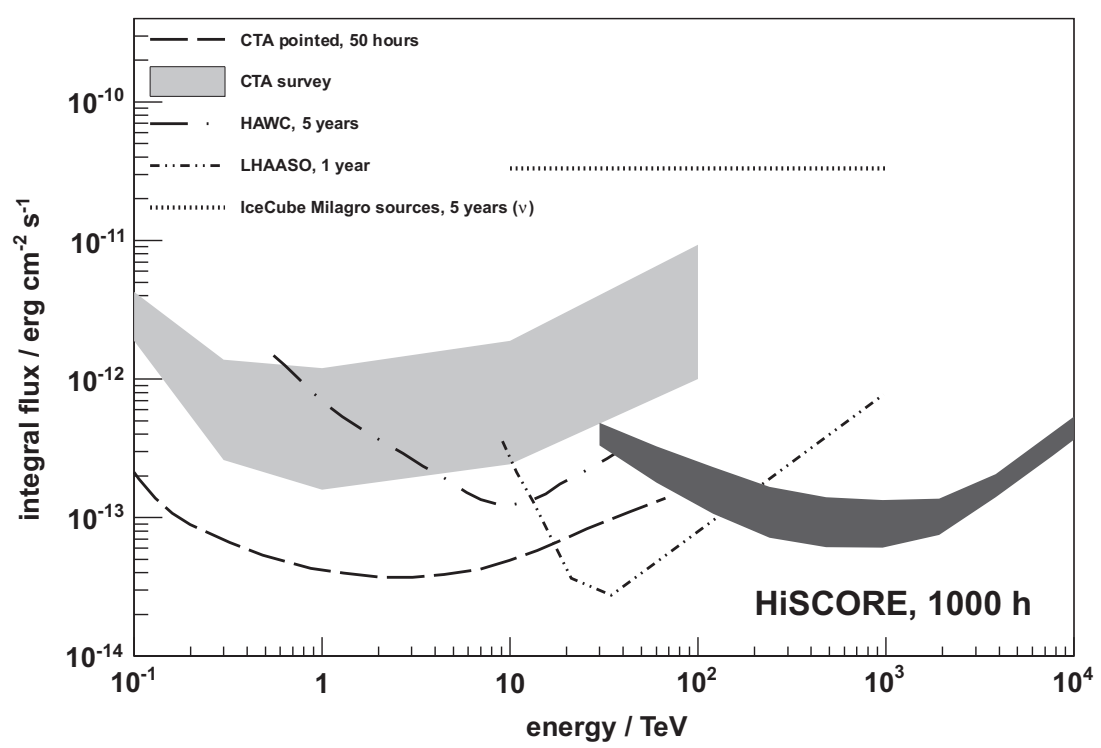

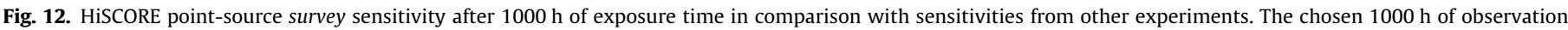

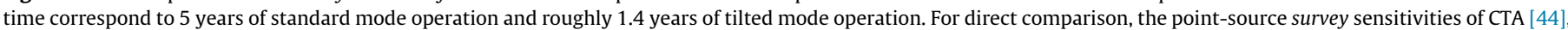

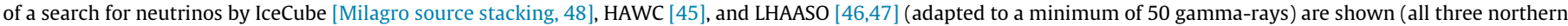
hemisphere). For reference, also the $50 \mathrm{~h}$ pointed-observation sensitivity of CTA [11] is given.

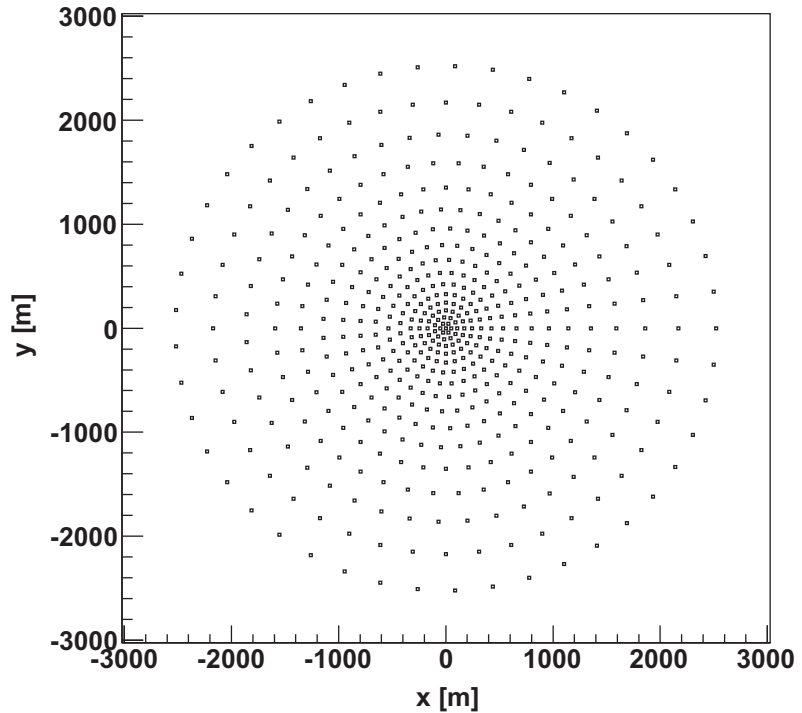

Fig. 13. Graded array layout (b). Using a comparable number of stations (493 as compared to 484 for the standard layout), the graded geometry optimizes the detection and reconstruction efficiency at low energies in the dense core of the array, and optimizes the sensitivity to large energies due to the larger area covered.

\section{Alternative array configurations}

For comparison to the standard array geometry and station configuration presented above, two further configurations were tested using the same simulation framework.

\subsection{Alternative configuration (a) $-12^{\prime \prime}$ PMTs}

This configuration consists of a standard geometry array configuration equipped with $12^{\prime \prime}$ PMTs and correspondingly larger Winston cones, resulting in a 2.25-fold increase in light collection area per station. As can be expected, the usage of larger PMTs allows to detect lower Cherenkov photon densities on the ground, therewith lowering the energy threshold of the detector array for air showers, i.e. maximizing the effective area at low energies. For this array configuration, a higher individual station threshold (factor 1.5) was used to limit the expected data rate to a level comparable to the expected rate for the standard array.

\subsection{Alternative configuration (b) - graded array}

A circular graded array layout of 493 stations with a dense core and inter-station distances gradually decreasing towards the edge of the array is shown in Fig. 13. At a comparable cost in number of stations, this layout results in improved effective areas at low energies (small inter-station distances in the array core) and at the same time optimizes the area at large energies due to the much larger area covered by the total array.

\subsection{Performance of alternative arrays}

The resulting effective gamma-ray trigger areas for these configurations are shown in Fig. 14. The described maximization of the effective area is clearly visible for configuration (a) at low energies and configuration (b) over the full energy range.

A comparison of the angular resolutions for both alternative configurations and the standard configuration is shown in Fig. 15. Configuration (a) shows an improvement of the angular resolution primarily at low energies, where the larger light collecting area of the $12^{\prime \prime}$ PMT stations improves the signal to noise ratio in each station and, more importantly, increases the number of stations per shower event, the stations being more sensitive to low light levels, i.e. larger core distances (also see Fig. 1). Towards high energies the latter advantage becomes less significant because the relative increase in number of stations is less strong for large shower events. The dense core of the graded array layout (b) also leads to a larger number of stations at lower energies (a larger number of stations being located inside the inner Cherenkov light pool, also see Fig. 1), i.e. an improved angular resolution as compared to the standard configuration. However, at higher energies $(E>330 \mathrm{TeV})$ a slight deterioration as compared to the standard configuration is seen. This can be explained by the fact that most 


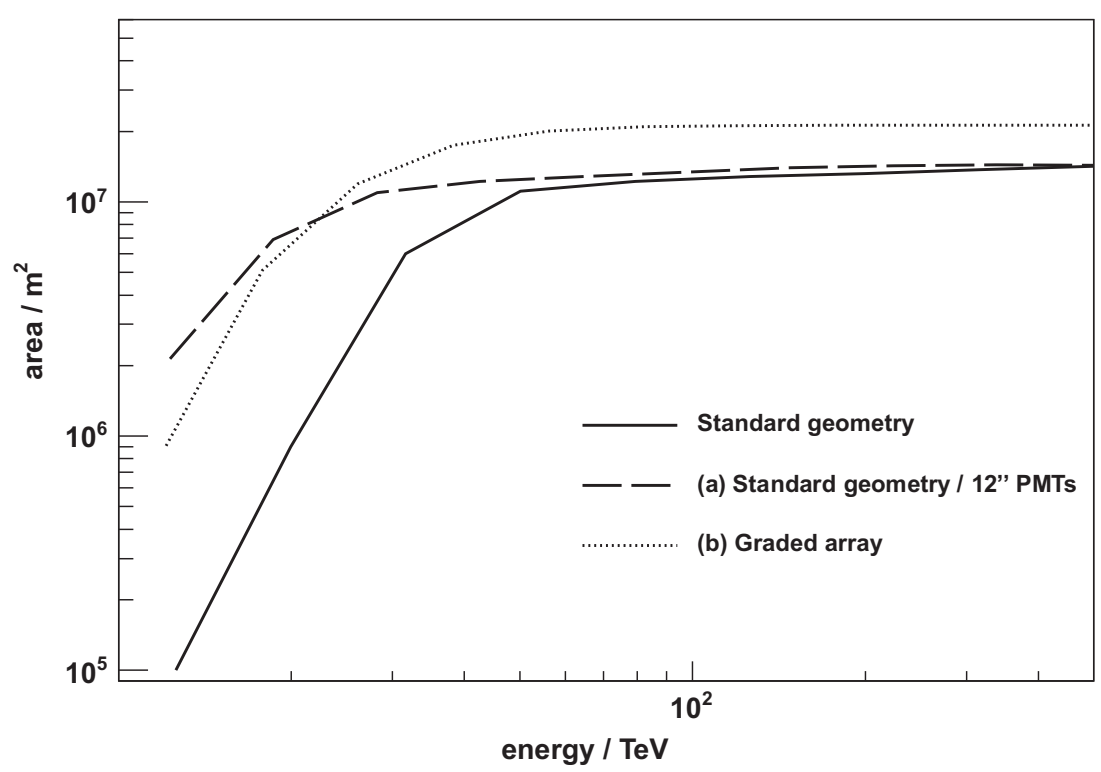

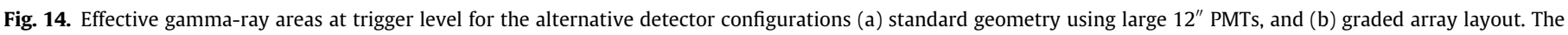
effective area for the standard configuration is shown for comparison (solid line).

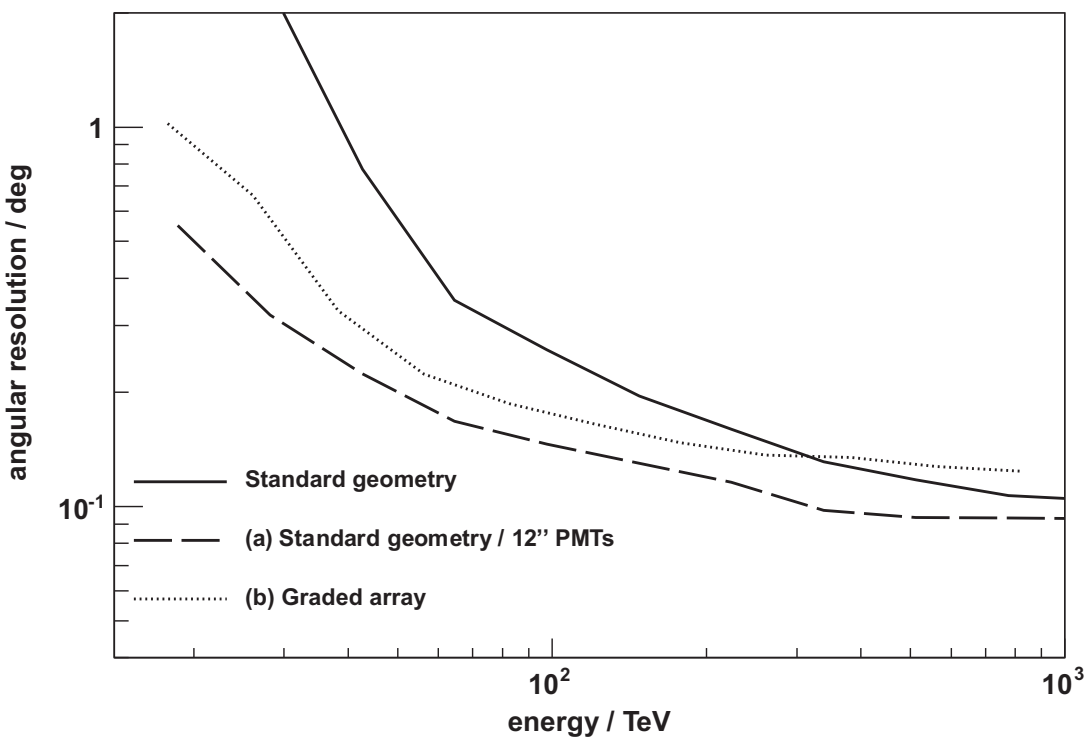

Fig. 15. Angular resolution ( 1 ns time jitter) for gamma-rays using the standard configuration, and the alternative configurations (a) and (b).

events at higher energies are detected by the low-density part of the array, with fewer stations per shower event than in the standard configuration.

Adaptations of the reconstruction (especially gamma-hadron separation) to different detector configurations are work in progress and will be the subject of a future publication. Quite obviously, a combination of a graded array also equipped with large PMTs would further improve the performance as compared to configurations (a) and (b) alone. Here, we aim at showing the effect of both alterations to the standard layout separately, for clarity.

\section{Outlook}

More complex array layouts, such as combining partly large PMTs with a graded design or different variants of graded designs, are under study. Moreover, for a further improvement at low energies, a cell solution is envisaged in which the four individual PMT channels are separated by $5-10 \mathrm{~m}$, building small sub-arrays (cells). Such cells could provide a better sampling of the central part of the LDF, and better reconstruction (thus sensitivity) at low energies ( $10 \mathrm{TeV}$ to $100 \mathrm{TeV})$. Simulations have shown that a cell solution can lower the energy threshold (defined as the energy at which the effective area reaches $50 \%$ of the instrumented area) considerably [26]. An extension of the concept we studied so far is to equip the underside of each sliding lid of the station boxes with scintillator material. With such a setup, continuation of data taking during daytime would allow to use HiSCORE as a very large charged particle shower front sampling array, effectively increasing the duty cycle by a factor of 9. Finally, we are also studying the benefits of a combination of the non-imaging technique simulated here with the imaging technique. 


\section{Summary}

We have described the HiSCORE concept for a large-area wideangle air shower experiment, based on an array of non imaging light collecting detector stations. A comprehensive simulation of the detector was performed including all relevant components (atmosphere, light-collection with Winston cones, photomultiplier, pulse shaping with afterpulses, station trigger). The resulting effective areas for various primary particles and the expected trigger rates for background have been calculated for different assumptions on the trigger threshold. The complete Monte Carlo simulation of the HiSCORE detector concept shows that such a non-imaging air-Cherenkov detector will be sufficiently sensitive to survey a large fraction ( $\pi \mathrm{sr}$ ) of the sky for gamma-ray sources above $10-50 \mathrm{TeV}$ (depending on the final array layout) at an energy flux level of a few $10^{-13} \mathrm{ergs} / \mathrm{cm}^{2}$ s. This sensitivity is comparable to the planned next-generation Cherenkov Telescope Array (CTA) at lower energies, effectively extending the sensitive energy range into the UHE gamma-ray regime. Furthermore, HiSCORE will provide high-statistics measurements of cosmic ray spectra and composition above $100 \mathrm{TeV}$ primary energy, covering the energy range of transition between Galactic and extragalactic origin of cosmic rays, and up to $10^{18} \mathrm{eV}$.

An engineering array with $1-2 \mathrm{~km}^{2}$ is planned for deployment 2014/2015, aiming at proof-of-principle measurements and first physics results.

\section{Acknowledgments}

We gratefully acknowledge valuable discussions on physics and hardware aspects with F. Aharonian, R. Antonini, N. Budnev, E. Lorenz, B.K. Lubsandorzhiev, R. Mirzoyan, G.P. Rowell, F. Schröder, T. Schweitzer, and M. Teshima. This research has made use of NASA's Astrophysics Data System Bibliographic Services. We acknowledge the support of the Helmholtz Russia Joint Research Group (HRJRG303). We acknowledge funding for the initiation of International Cooperation (TL 51-3) by the Deutsche Forschungsgemeinschaft, DFG, and the Russian RFFI (13-02-12095). Furthermore, we acknowledge funding provided by the Helmholtz Gemeinschaft, Germany in the framework of the Helmholtz Alliance for Astroparticle Physics (HAP).

\section{Appendix A. Pulse shape parametrization}

The single photo-electron response of a PMT is determined by the pulse shape as a function of time, and a probability distribution of the amplitude response including afterpulses. Here, we used a probability for a response amplitude at 4 p.e. of $10^{-4}$. The normalized pulse shape $\left(R_{\text {shape }}(t)\right)$ used in this work was taken from Henke [30]. This shape was used to describe the response of AIROBICC, which was using the same PMT as envisaged for HiSCORE. The pulse shape is shown as inlay in Fig. 5. It can be described as

$R_{\text {shape }}(t)=D \cdot t^{\prime a} \cdot e^{\left(-b t^{\prime}\right)^{c}}$

where $D$ is a normalization constant, $a=1.25, b=0.0414, c=$ 1.48 , and the time $t$ (in ns) is included in $t^{\prime}=\frac{7}{4} t / 1 \mathrm{~ns}$. The response function $R_{\text {shape }}(t)$ is a parametrization of the normalized, dimensionless single photo-electron response function. The parameters $a, b$, and $\mathrm{c}$ are dimension-less as well.

\section{References}

[1] T. Antoni, W.D. Apel, A.F. Badea, et al., Search for cosmic-ray point sources with KASCADE, Astrophys. J. 608 (2004) 865.

[2] K. Kotera, A.V. Olinto, The astrophysics of ultrahigh energy cosmic rays, Annu. Rev. Astron. Astrophys. 49 (2011) 119.
[3] P. Baillon, L. Behr, S. Danagoulian, THEMISTOCLE Collaboration, Gamma ray spectrum of the Crab nebula in the multi TeV region k20, Astropart. Phys. 1 (1993) 341.

[4] A. Karle, M. Merck, R. Plaga, et al., Design and performance of the angle integrating Cerenkov array AIROBICC, Astropart. Phys. 3 (1995) 321.

[5] J.W. Fowler, L.F. Fortson, C.C.H. Jui, et al., A measurement of the cosmic ray spectrum and composition at the knee, Astropart. Phys. 15 (2001) 49.

[6] S.F. Berezhnev, D. Besson, N.M. Budnev, et al., The Tunka-133 EAS Cherenkov light array: status of 2011, Nucl. Instrum. Methods Phys. Res. A 692 (2012) 98

[7] M.N. Dyakonov, S.P. Knurenko, V.A. Kolosov, et al., The use of Cherenkov detectors at the Yakutsk cosmic ray extensive air shower array, Nucl. Instrum. Methods Phys. Res. A 248 (1986) 224.

[8] T. Huege, W.D. Apel, J.C. Arteaga, T. Asch, et al., The LOPES experiment - recent results, status and perspectives, Nucl. Instrum. Methods Phys. Res. A 662 (2012) 72.

[9] P.W. Gorham, N.G. Lehtinen, G.S. Varner, et al., Observations of microwave continuum emission from air shower plasmas, Phys. Rev. D 78 (2008) 032007.

[10] R. Šmída, S. Baur, M. Bertaina, et al., Observation of microwave emission from extensive air showers with CROME, Eur. Phys. J. Web Conf. 53 (2013) 8010.

[11] M. Actis, G. Agnetta, F. Aharonian, A. Akhperjanian, et al., CTA Consortium, Design concepts for the Cherenkov Telescope Array CTA: an advanced facility for ground-based high-energy gamma-ray astronomy, Exp. Astron. 32 (2011) 193.

[12] G. Rowell, V. Stamatescu, R. Clay, et al., Tenten: a new IACT array for multi-TeV gamma-ray astronomy, Nucl. Instrum. Methods Phys. Res. A 588 (2008) 48.

[13] S. Gabici, F.A. Aharonian, Searching for Galactic cosmic-ray pevatrons with Multi-TeV gamma rays and neutrinos, Astrophys. J. Lett. 665 (2007) L131.

[14] M. Tluczykont, D. Hampf, D. Horns, et al., The ground-based large-area wideangle $\gamma$-ray and cosmic-ray experiment HiSCORE, Adv. Space Res. 48 (2011) 1935.

[15] S. Ritt, R. Dinapoli, U. Hartmann, Application of the DRS chip for fast waveform digitizing, Nucl. Instrum. Methods Phys. Res. A 623 (2010) 486.

[16] J. Serrano, P. Alvarez, M. Cattin, E.G. Cota, P.M.J.H. Lewis, T. Wlostowski, et al., The white rabbit project, in: Proceedings of ICALEPCS TUC004, Kobe, Japan. Available at: <http://www.ohwr.org/projects/white-rabbit/>.

[17] M.Brueckner, R.Wischnewski, A white rabbit setup for sub-nsec synchronization, timestamping and time calibration in large scale astroparticle physics experiments, in: Proceedings of the 33rd ICRC, Rio de Janeiro, paper 1146 , in press.

[18] M. Brueckner, R. Wischnewski, S. Berezhnev, et al., Results from the whiterabbit sub-nsec time synchronization setup at HiSCORE-Tunka, in: Proceedings of the 33rd ICRC, Rio de Janeiro, paper 1158, in press.

[19] R. Wischnewski, S. Berezhnev, M. Brueckner, et al., Status of the HiSCORE experiment, paper 1164 , in press.

[20] N.M. Budnev, O.B. Chvalaev, O.A. Gress, Data acquisition system for the TUNKA-133 Array, Astroparticle, Particle and Space Physics, Detectors and Medical Physics Applications (2008) 287

[21] F.G. Schröder, T. Asch, L. Bähren, et al., New method for the time calibration of an interferometric radio antenna array, Nuc. Instrum. Methods Phys. Res. A 615 (2010) 277.

[22] D. Heck, T. Pierog, J. Knapp, CORSIKA: an air shower simulation program, Astrophys. Source Code Library (2012).

[23] N.N. Kalmykov, S.S. Ostapchenko, A.I. Pavlov, Quark-gluon-string model and EAS simulation problems at ultra-high energies, Nuc. Phys. B Proc. Suppl. 52 (1997) 17.

[24] H. Fesefeldt, Report PITHA-85/02, RWTH Aachen, 1985.

[25] T. Pierog, LHC data and extensive air showers, Eur. Phys. J. Web Conf. 52 (2013) 03001.

[26] D. Hampf, M. Tluczykont, D. Horns, Simulation of the expected performance for the proposed gamma-ray detector HiSCORE, in: Proceedings of Science (Texas 2010), 2010, p. 245.

[27] K. Bernlöhr, Simulation of imaging atmospheric Cherenkov telescopes with CORSIKA and sim_telarray, Astropart. Phys. 30 (2008) 149.

[28] D. Hampf, Study for the wide-angle air cherenkov detector HiSCORE and time gradient event reconstruction for the H.E.S.S. experiment (PhD thesis), University of Hamburg, 2012. <http://ediss.sub.uni-hamburg.de/volltexte/ 2012/5699/>.

[29] F. Kneizys, L. Abreu, G. Anderson, et al., in: L.W. Abreu, G.P. Anderson (Eds.) The modtran 2/3 report and lowtran 7 model, 1996.

[30] V. Henke, Studie zur Auswertung der Ankunftszeitverteilung des CerenkovLichts ausgedehnter Luftschauer. (Diploma thesis), University of Hamburg, 1994.

[31] J.R. Hörandel, On the knee in the energy spectrum of cosmic rays, Astropart Phys. 19 (2003) 193.

[32] D. Hampf, G. Rowell, N. Wild, T. Sudholz, D. Horns, M. Tluczykont, Measurement of night sky brightness in southern Australia, Adv. Space Res. 48 (2011) 1017.

[33] K. Nakamura et al., Particle data group, J. Phys. G 37 (2010) 075021.

[34] D. Hampf, M. Tluczykont, D. Horns, Event reconstruction techniques for the wide-angle air Cherenkov detector HiSCORE, Nucl. Instrum. Methods Phys. Res. A 712 (2013) 137.

[35] T.-P. Li, Y.-Q. Ma, Analysis methods for results in gamma-ray astronomy, Astrophys. J. 272 (1983) 317.

[36] F. Aharonian, A. Akhperjanian, K.-M. Aye, et al., The H.E.S.S. survey of the inner Galaxy in very high-energy gamma-rays, Astrophys. J. 636 (2006) 777.

[37] B. Bartoli, P. Bernardini, X.J. Bi, et al., TeV gamma-ray survey of the northern sky using the ARGO-YBJ detector, Astrophys. J. 779 (2013) 27. 
[38] A.A. Abdo, B. Allen, D. Berley, et al., Discovery of TeV gamma-ray emission from the cygnus region of the Galaxy, Astrophys. J. 658 (2007) L33.

[39] M. Meyer, D. Horns, H.-S. Zechlin, The Crab Nebula as a standard candle in very high-energy astrophysics, Astron. Astrophys. 523 (2010) A2.

[40] A.A. Abdo, B. Allen, D. Berley, et al., TeV gamma-ray sources from a survey of the Galactic plane with Milagro, Astrophys. J. 664 (2007) L91.

[41] F. Aharonian, A.G. Akhperjanian, G. Anton, et al., Detection of very high energy radiation from HESS J1908+063 confirms the Milagro unidentified source MGRO J1908+06, Astron. Astrophys. 499 (2009) 723.

[42] B. Bartoli, P. Bernardini, X.J. Bi, et al., Observation of the TeV gamma-ray source MGRO J1908+06 with ARGO-YBJ, Astrophys. J. 760 (2012) 110.

[43] M. Tluczykont, D. Horns, D. Hampf, R. Nachtigall, et al., HiSCORE: a new detector for astroparticle and particle physics beyond $10 \mathrm{TeV}$, Nucl. Instrum. Methods Phys. Res. A 692 (2012) 246.
[44] G. Dubus, J. Contreras, S. Funk, et al., Surveys with the Cherenkov Telescope Array, Astropart. Phys. 43 (2013) 317.

[45] G. Sinnis, HAWC: a next generation VHE all-sky telescope, in: F.A. Aharonian, H.J. Völk, D. Horns (Eds.), Heidelberg Gamma-Ray Symposium, American Institute of Physics, vol. 745, p. 234.

[46] Z. Cao, A future project at Tibet: the large high altitude air shower observatory (LHAASO), Chin. Phys. C 34 (2010) 249.

[47] S. Cui, Y. Liu, Y. Liu, X. Ma, Simulation on gamma ray astronomy research with LHAASO-KM2A, Astropart. Phys. 54 (2014) 86-92.

[48] M.C. Gonzalez-Garcia, F. Halzen, S. Mohapatra, Identifying Galactic PeVatrons with neutrinos, Astropart. Phys. 31 (2009) 437. 